\title{
Strong Short-Range Magnetic Order in a Frustrated FCC Lattice and Its Possible Role in the Iron Structural Transformation
}

\author{
A. N. Ignatenko ${ }^{a}$, A. A. Katanin ${ }^{a, b}$, and V. Yu. Irkhin ${ }^{a}$ \\ ${ }^{a}$ Institute of Metal Physics, Ural Division, Russian Academy of Sciences, Yekaterinburg, 620041 Russia \\ e-mail: ignatenko@imp.uran.ru \\ ${ }^{b}$ Max-Planck-Institut für Festkörperforschung, 70569 Stuttgart, Germany
}

Received April 9, 2008

\begin{abstract}
The magnetic properties of a frustrated Heisenberg antiferromagnet with the fcc lattice and exchange interaction between the nearest $\left(J_{1}\right)$ and next-to-nearest $\left(J_{2}\right)$ neighbors are studied in this work. For the collinear phase with the wave vector $\mathbf{Q}=(\pi, \pi, \pi)$, the equations of the self-consistent spin-wave theory are obtained and solved numerically for the sublattice magnetization and the averaged short-range order parameter. The dependence of the Néel temperature $T_{\mathrm{N}}$ on the ratio $J_{2} / J_{1}$ is found. It is shown that, in the case of a sufficiently strong frustration, strong short-range magnetic order persists over a wide temperature range above $T_{\mathrm{N}}$. The possible application of this result to the mechanism of structural phase transition from $\alpha$-Fe to $\gamma$-Fe is considered.
\end{abstract}

PACS numbers: 75.10.Jm, 75.30.Kz, 75.50.Bb

DOI: $10.1134 / \mathrm{S} 0021364008100093$

The short-range magnetic order (SRMO) can play a crucial role in the thermodynamic properties near the magnetic phase transitions. This is also true for the structural phase transitions, because the presence of a more pronounced short-range order in one of the phases (even in the absence of the long-range order) can influence the nucleation of a new phase when changing the temperature, pressure, or impurity concentration. In this connection, of particular interest are the magnetic quantum phase transitions (MQPTs) occurring at temperature $T=0$ and at a certain ratio between the physical parameters (exchange integrals, pressure, impurity concentration, etc.). Inasmuch as the long-range order parameter is small and the transition temperature is low near the MQPT, the strong short-range order can persist over a wide temperature range and influence the structural transformations.

Such a situation is expected, in particular, for iron. Iron is known to exist in two, bcc $(\alpha-\mathrm{Fe})$ and fcc $(\gamma-\mathrm{Fe})$, crystal modifications. The low-temperature bcc phase is ferromagnetic at $T<T_{\mathrm{C}}=1045 \mathrm{~K}$. At $T=T_{\mathrm{S}}=$ $1185 \mathrm{~K}$, the structural transition occurs to the $\gamma$ phase, which is stable in the temperature interval on the order of $200 \mathrm{~K}$ above $T_{\mathrm{S}}$. With a further increase in temperature, iron transforms to the high-temperature bcc phase. There is an assumption that the structural phase transition from $\alpha$ to $\gamma$ iron is caused by the presence of a strong short-range antiferromagnetic order in the $\gamma$ phase. Contrary to the macroscopic three-dimensional samples, where the $\gamma$ phase occurs only at high temperatures in a magnetically disordered state, the $\gamma$ phase in the granular form is stable at low temperatures and transforms to the antiferromagnetic state at the temper- ature $T_{\mathrm{N}} \sim 100 \mathrm{~K}$ [1]. Although the Néel temperature is low (compared to the ferromagnetic transition temperature in the bcc phase), a strong short-range antiferromagnetic order can occur at $T \gg T_{\mathrm{N}}$. Experimentally, the SRMO is manifested by the presence of the narrow neutron inelastic-scattering lines and a well-defined spin-wave spectrum in the magnetically disordered phase.

Recent theoretical studies of the SRMO in the twodimensional [2] and quasi-two-dimensional [3] systems have shown that it can persist up to the temperatures $T_{\mathrm{N}} \ll T \lesssim J$ ( $J$ is the exchange parameter). A particularly broad temperature range is expected for the SRMO near the frustration point (where the competition between the different magnetic phases is the strongest). The short-range order in a frustrated system was studied only for the square and cubic lattices with the exchange between the nearest and next-to-nearest neighbors [4]. In the strong-frustration regime, the state without long-range magnetic order (spin liquid) exists in the square lattice at $T=0$ [5]. An analogous state can also occur in the fcc lattice. It is thus of interest to investigate magnetic properties of a three-dimensional system with the fcc lattice near the frustration point, where the strong SRMO is expected at temperatures well above $T_{\mathrm{N}}$.

It is convenient to study SRMO using the self-consistent spin-wave theory (SSWT) [2-4], because the short-range order parameters $\gamma_{\delta}=\sqrt{\left|\left\langle\mathbf{S}_{i} \cdot \mathbf{S}_{i+\delta}\right\rangle\right|} / S\left(\mathbf{S}_{i}\right.$ is the operator of spin $S$ on the site $i$, and $\delta$ stands for the vector connecting two neighboring sites) appear in a natural way after decoupling of the quaternary Bose- 


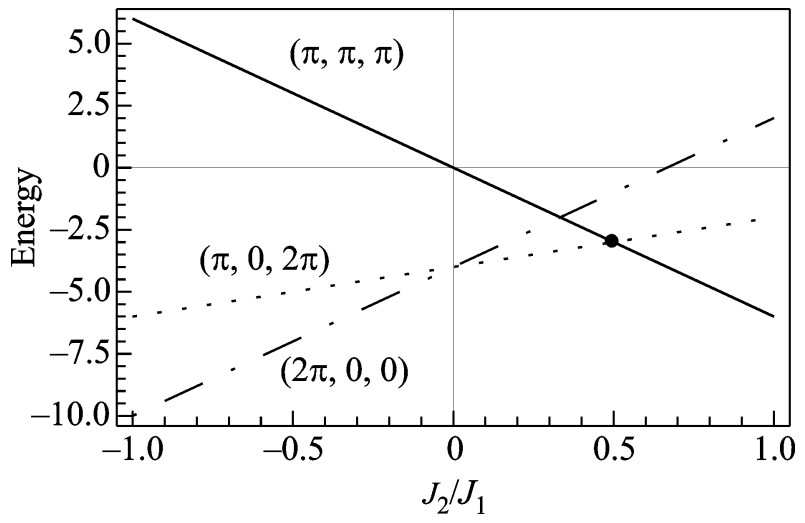

Fig. 1. Energies of different classical spin configurations as functions of the ratio $r=J_{2} / J_{1}$.

operator products describing the magnon-magnon interaction. This theory is based on the spin-wave spectrum that is observed experimentally over a wide temperature range above the Néel temperature in the systems with low $T_{\mathrm{N}}$. In contrast to the standard spin-wave theory (SWT), the SSWT is also operative in the disordered phase and includes both the temperature and quantum renormalizations of SRMO.

The spin subsystem is modeled by the quantum Heisenberg Hamiltonian on the fcc lattice:

$$
H=\frac{1}{2} \sum_{i j} J_{i j} \mathbf{S}_{i} \cdot \mathbf{S}_{j}
$$

with the interactions $J_{1}$ and $J_{2}$ between the nearest and next-to-nearest neighbors, respectively. In the limit $S \longrightarrow \infty$, the Hamiltonian reduces to its classical form. The energies of the possible configurations are given in Fig. 1 as functions of the parameter $r=J_{2} / J_{1}\left(J_{1}>0\right)$. At $r>1 / 2$, the collinear structure

$$
\left\langle\mathbf{S}_{i}\right\rangle=(-1)^{\mathbf{Q} \cdot \mathbf{R}_{i}} \mathbf{M}
$$

with the wave vector $\mathbf{Q}=(\pi, \pi, \pi)$ is stable (lattice parameter $a=1)$. In Eq. (2), $\mathbf{M}$ is the sublattice-magnetization vector and $\mathbf{R}_{i}$ is the radius vector of the $i$ th lattice site. In the interval $0<r<1 / 2$, the ground state with the wave vector $(\pi, 0,2 \pi)$ becomes noncollinear:

$$
\left\langle\mathbf{S}_{i}\right\rangle=\mathbf{M}_{1} \cos \mathbf{Q} \cdot \mathbf{R}_{i}+\mathbf{M}_{2} \sin \mathbf{Q} \cdot \mathbf{R}_{i},
$$

where $\mathbf{M}_{1}$ and $\mathbf{M}_{2}$ are the mutually orthogonal vectors of the same length. At $r<0$, the ground state with the wave vector $(2 \pi, 0,0)$ again becomes collinear. In the limit $S \longrightarrow \infty$, all transitions between these phases are of the first order. In the presence of quantum fluctuations, the point $r=1 / 2$ (indicated in the figure) becomes the frustration point [6]. In contrast to the point $r=1 / 2$, the quantum corrections in the vicinity of the phase- transition point ( $r=0, T=0)$ do not qualitatively affect the phase diagram, and the spin-liquid phase does not appear.

To examine the role of the quantum and temperature fluctuations near the frustration point in the collinear $\mathbf{Q}=(\pi, \pi, \pi)$ phase, it is convenient to use the Bar'yakhtar-Krivoruchko-Yablonskii representation and write the spin operators through the Bose and Fermi operators $b_{i}$ and $c_{i}$ [7]:

$$
\begin{gathered}
S_{i}^{z}=(-1)^{\mathrm{Q} \cdot \mathbf{R}_{i}}:\left(S-B_{i}^{\dagger} B_{i}-(2 S+1) c_{i}^{\dagger} c_{i}\right):, \\
S_{i}^{+}=\sqrt{2 S} B_{i}, \\
S_{i}^{-}=\sqrt{2 S}:\left(B_{i}^{\dagger}-\frac{1}{2 S} B_{i}^{\dagger} B_{i}^{\dagger} B_{i}\right):-\frac{2(2 S+1)}{\sqrt{2 S}} B_{i}^{\dagger} c_{i}^{\dagger} c_{i},
\end{gathered}
$$

where $B_{i}=b_{i}$ for one sublattice and $B_{i}=b_{i}^{\dagger}$ for the other. The symbol :: stands for the normal ordering.

The standard SSWT variant $[2,3]$ can be obtained by inserting expressions (4) into the Hamiltonian followed by the Hartree-Fock decoupling of the arising quaternary terms. For the $\mathbf{Q}=(\pi, \pi, \pi)$ phase, this procedure faces certain problems associated with the "order from disorder" phenomenon [8]. The matter is that, in the limit $S \longrightarrow \infty$, the ground state of Hamiltonian (1) is strongly degenerate at $r>1 / 2$. This state represents a system of four simple cubic sublattices enclosed in one another, with the staggered antiferromagnetic order and independent directions of the Néel magnetization in each of them. In the standard SWT, new gapless modes corresponding to the rotation of the sublattice Néel vectors relative to one another appear in the Brillouin zone at $T<T_{\mathrm{N}}$ in addition to the standard Goldstone modes. However, after applying the $1 / S$ corrections to the SWT, "quantum" gaps open and broaden with the temperature at the points where the excess gapless modes are located. Such a behavior for the gaps is contradictory to the experiments with garnet $\mathrm{Ca}_{3} \mathrm{Fe}_{2} \mathrm{Ge}_{3} \mathrm{O}_{12}$, for which the situation is analogous [9]. The "erroneous" growth predicted for the quantum gaps by the standard SSWT greatly affects the thermodynamic behavior of the system, because it rules out frustration and leads to the strongly overstated value of $T_{\mathrm{N}}$, as compared to the SWT.

To resolve this problem, we use a variant of the SSWT with the averaged short-range order parameter, for which the quantum gaps are absent at any temperature. The averaged SRMO parameter is defined as [3]

$$
\bar{\gamma}=\left(\gamma_{1 A F}-\gamma_{1 F}+r \gamma_{2}\right) / r,
$$

where $\gamma_{1 A F}$ and $\gamma_{1 F}$ are, respectively, the SRMO parameters corresponding to the nearest antiferromagnetic and ferromagnetic neighbors and $\gamma_{2}$ is the SRMO parameter for the next-to-nearest antiferromagnetic neighbors. By inserting Eq. (4) into the Hamiltonian 


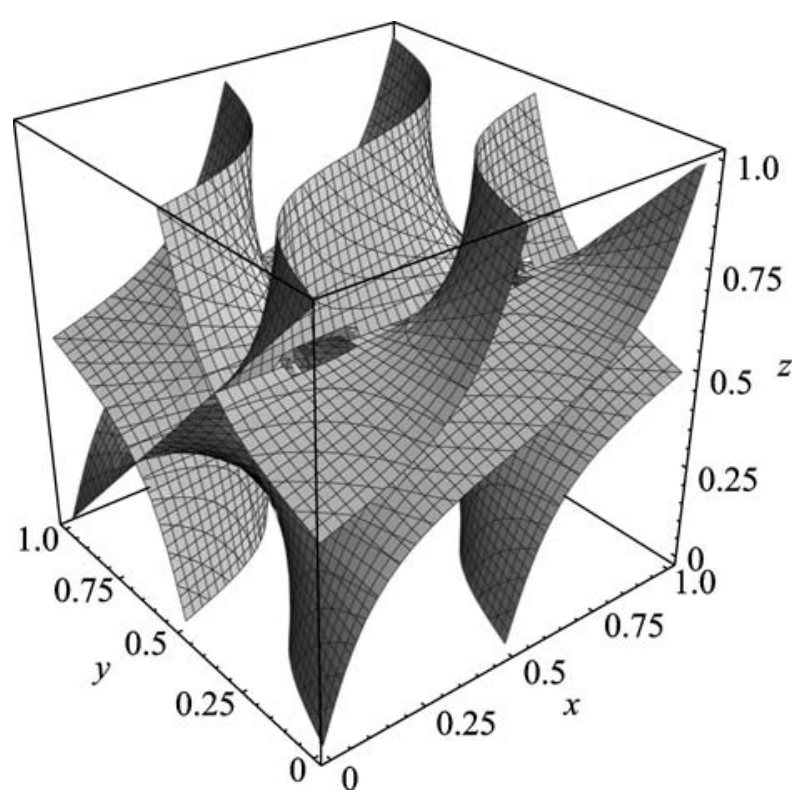

Fig. 2. Zero-energy surfaces in the Brillouin zone for $r=$ $J_{2} / J_{1}=1 / 2$ in the coordinates $x=\left(k_{x}+k_{y}\right) / 4 \pi, y=\left(k_{x}+\right.$ $\left.k_{z}\right) / 4 \pi$, and $z=\left(k_{z}+k_{y}\right) / 4 \pi$ varying from 0 to 1 .

and performing the Hartree-Fock decoupling in the resulting quaternary terms, one arrives at the following equations for the sublattice magnetization $\mathbf{M}$ and the parameter $\bar{\gamma}$ (hereafter $J_{1}=1$ ):

$$
\begin{aligned}
& M^{z}=(S+1 / 2) \operatorname{coth}\left[\frac{e_{0}(S+1 / 2)}{T}\right] \\
&-\frac{1}{2} \int \frac{d^{3} k}{(2 \pi)^{3}} \frac{P_{k} \operatorname{coth}\left(E_{k} / 2 T\right)}{E_{k}}, \\
& S \bar{\gamma}=(S+1 / 2) \operatorname{coth}\left[\frac{e_{0}(S+1 / 2)}{T}\right] \\
&+\frac{1}{2} \int \frac{d^{3} k}{(2 \pi)^{3}}\left(g_{k} \cos \frac{k_{x}+k_{y}}{2}\right. \\
&\left.+r g_{k} \cos k_{y}-P_{k} \cos \frac{k_{y}+k_{z}}{2}\right) \frac{\operatorname{coth}\left(E_{k} / 2 T\right)}{E_{k}},
\end{aligned}
$$

where $E_{k}=\sqrt{P_{k}^{2}-g_{k}^{2}}$ is the spectrum of the elementary excitations

$$
\begin{gathered}
e_{0}=6 \operatorname{Sr} \bar{\gamma}-\mu, \\
P_{k}=e_{0} \\
+2 S \bar{\gamma}\left(\cos \frac{k_{y}-k_{x}}{2}+\cos \frac{k_{y}+k_{z}}{2}+\cos \frac{k_{x}+k_{z}}{2}\right),
\end{gathered}
$$

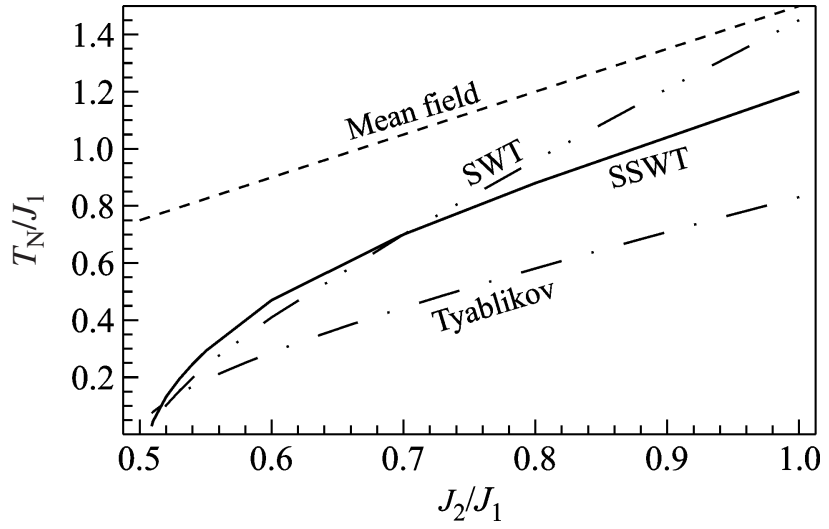

Fig. 3. Néel temperature versus $r=J_{2} / J_{1}$ in the SSWT, mean-field, Tyablikov, and SWT approximations.

$$
\begin{aligned}
g_{k}=2 S \bar{\gamma}\left(\cos \frac{k_{z}-k_{x}}{2}+\cos \frac{k_{z}-k_{y}}{2}+\cos \frac{k_{x}+k_{y}}{2}\right) \\
+2 S \bar{\gamma} r\left(\cos k_{x}+\cos k_{y}+\cos k_{z}\right) .
\end{aligned}
$$

The boson chemical potential $\mu$ is zero in the ordered phase and negative in the disordered phase, where it is determined by the equation $\left\langle S_{i}^{z}\right\rangle=0$ [2]. At the frustration point $r=1 / 2$, the spin-wave spectrum of the magnetically ordered state $(\mu=0)$ has the form

$$
\begin{aligned}
E_{k}= & 2 S \bar{\gamma}\left|\cos \frac{k_{x}}{2}+\cos \frac{k_{y}}{2}+\cos \frac{k_{z}}{2}\right| \\
& \times\left|\sin \frac{k_{x}}{2}+\sin \frac{k_{y}}{2}+\sin \frac{k_{z}}{2}\right| .
\end{aligned}
$$

This expression turns to zero at certain surfaces in the Brillouin zone (Fig. 2), as a result of which magnetization (6) formally tends to $-\infty$ at zero and finite temperatures. This is contradictory to the assumption of the magnetically ordered ground state and is indicative of the competition between the two magnetic phases $(\pi, \pi$, $\pi)$ and $(\pi, 0,2 \pi)$ resulting in a new phase without the long-range magnetic order (spin liquid) near the frustration point $r=1 / 2$. Note that, in contrast to the fcc lattice, the sublattice magnetization in the simple cubic (sc) lattice does not diverge formally at the frustration point at $T=0$ (but not at $T>0$ ), because the spin-wave spectrum turns to zero only on certain lines. Correspondingly, the solution to the SSWT equations with the averaged order parameter suggests that the spin-liquid state in the sc lattice is possible only for $S=1 / 2$, whereas in the fcc lattice it can occur for any $S$.

The temperature domain of existence of the shortrange order was determined by numerically solving Eqs. (6) and (7) for spin $S=1 / 2$ (Figs. 3-5). The $r$ dependences of the Néel temperature, as obtained in the SSWT, mean-field, Tyablikov, and SWT approximations, are demonstrated in Fig. 3. The mean-field theory 


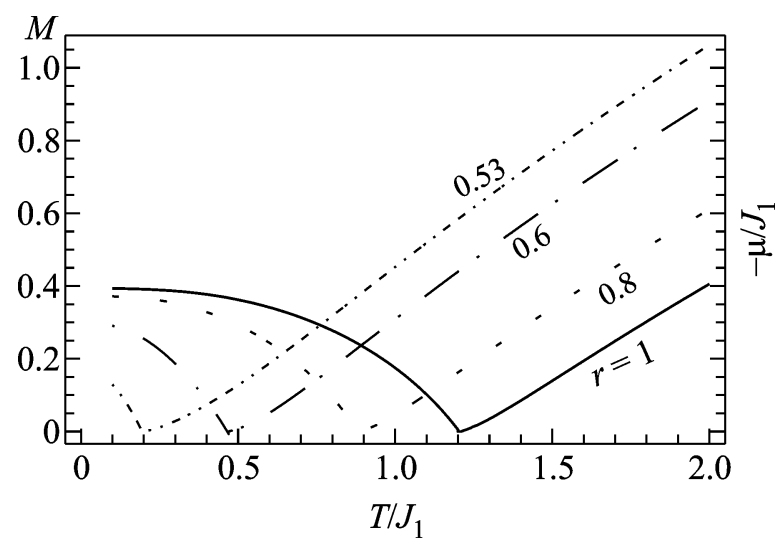

Fig. 4. Temperature dependences of the sublattice magnetization at $T<T_{\mathrm{N}}$ and the chemical potential $\mu$ at $T>T_{\mathrm{N}}$ for different $r$.

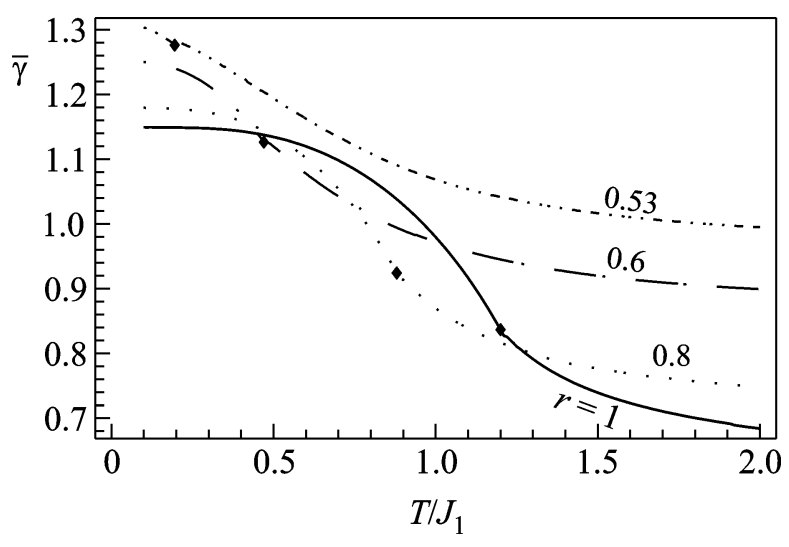

Fig. 5. Temperature dependence of the averaged SRMO parameter for different $r$. The dots indicate the Néel temperature $T_{\mathrm{N}} / J_{1}$.

the experimental determination of these parameters also faces problems [13], it allows the hypothesis of the proximity of $\gamma$-Fe to the frustration point to be either justified or ruled out.

The approach developed in this work can further be used in the description of the Fe $\gamma-\alpha$ transition induced by the magnetic fluctuations. Due to the strong SRMO, the structural fcc-bcc transition can occur at temperatures far above $T_{\mathrm{N}}$, in compliance with the experimental data on $T_{\mathrm{S}}$, and proceed by a purely magnetic mechanism. The Heisenberg model with strain can be used to more precisely formulate the qualitative picture and quantitatively describe the structural phase transition under discussion.

We are grateful to Yu.N. Gornostyrev for discussion and valuable remarks. This work was supported by the Russian Foundation for Basic Research (project no. 0702-01264a), the Council of the President of the Russian Federation for Support of Young Scientists and Leading Scientific Schools (project no. NSh-4640.2006.2), and the OAO MMK, ITTs "Ausferr," and FGiO "Intels" (project no. 49-07-01).

\section{REFERENCES}

1. S. C. Abrahams, L. Guttman, and J. S. Kasper, Phys. Rev. 127, 2052 (1962); G. J. Johanson, M. B. McGirr, and D. A. Wheeler, Phys. Rev. B 1, 3208 (1970); Y. Tsunoda, N. Kunitomi, and R. M. Nicklow, J. Phys. F: Met. Phys. 17, 2447 (1987).

2. M. Takahashi, Phys. Rev. B 40, 2494 (1989); D. J. Yoshioka, Phys. Soc. Jpn. 58, 3733 (1989).

3. V. Yu. Irkhin, A. A. Katanin, and M. I. Katsnelson, Phys. Rev. B 60, 1082 (1999); A. A. Katanin and V. Yu. Irkhin, Usp. Fiz. Nauk 177, 639 (2007) [Phys. Usp. 50, 613 (2007)].

4. V. Yu. Irkhin, A. A. Katanin, and M. I. Katsnelson, J. Phys.: Cond. Matter 4, 5227 (1992). 
5. O. P. Sushkov, J. Oitmaa, and Zheng Weihong, Phys. Rev. B 63, 104420 (2001); J. Sirker, Zheng Weihong, O. P. Sushkov, and J. Oitmaa, Phys. Rev. B 73, 184420 (2006).

6. T. Yildirim, A. B. Harris, and E. F. Shender, Phys. Rev. B 58, 3144 (1998).

7. V. G. Bar'yakhtar, V. N. Krivoruchko, and D. A. Yablonskii, Green's Functions in the Theory of Magnetism (Naukova Dumka, Kiev, 1984) [in Russian].

8. A. M. Tsvelik, Quantum Field Theory in Condensed Matter Physics (Cambridge Univ. Press, Cambridge, 1998).

9. Th. Bruckel, B. Dorner, A. Gukasov, and V. Plakhty, Phys. Lett. A 162, 357 (1992).
10. V. A. Gubanov, A. I. Liechtenstein, and A. V. Postnikov, Magnetic and the Electronic Structure of Crystals (Springer, Berlin, Heidelberg, New York, 1992).

11. O. N. Mryasov et al., J. Phys.: Cond. Matter 3, 7683 (1991); D. W. Boukhvalov, Yu. N. Gornostyrev, M. I. Katsnelson, et al., Phys. Rev. Lett. 99, 247205 (2007).

12. A. V. Ruban, M. I. Katsnelson, W. Olovsson, et al., Phys. Rev. B 71, 054402 (2005).

13. P. Böni, G. Shirane, and J. P. Wicksted, Phys. Rev. B 31, 4597 (1985).

Translated by V. Sakun 